\title{
Elective Intermittent Temporary Clipping in Aneurysm Surgery: A Practical Protocol
}

\author{
S. Selvapandian ${ }^{1} \quad$ P. Sai Sudarsan ${ }^{1} \quad$ Pannakal G. Shaji $^{2}$ Mathew J. Chandy ${ }^{3}$ \\ ${ }^{1}$ Department of Neurosurgery, Apollo Speciality Hospital, \\ Vanagaram, Chennai, India \\ 2 Sunrise Hospital, Kakkanad, Kochi, Kerala \\ Address for correspondence Dr. S. Selvapandian, MCh, Department \\ of Neurosurgery, Apollo Specialty Hospital, Vanagaram, Chennai, \\ India (e-mail: sselvapandian@gmail.com).
}

${ }^{3}$ Department of Neurosurgery, Apollo Hospital, Dhaka, Bangladesh

Indian J Neurosurg 2015;4:8-14.

\begin{abstract}
Background Temporary occlusion of the proximal vessel is being increasingly used as an adjunct during aneurysm surgery for safer dissection, evacuation of the aneurysm, and to prevent intraoperative rupture. The guidelines regarding its practice are ambiguous. One of the major concerns is the safe time limit for temporary occlusion. Studies done so far have failed to reach a consensus on this issue. This study is an attempt to provide a practical, safe time period of intermittent occlusion of an artery in the anterior circulation, irrespective of vascular territory.

Patients and Methods A total of 68 patients in whom elective, intermittent, temporary clipping of the proximal vessel was employed during aneurysm surgery were prospectively studied. They were divided into two groups, Group I with an occlusion period of less than 7 minutes and Group II with occlusion period of 10 minutes and above per episode. The incidence of complications in both groups, their relationship to the number of intermittent clippings, and the total duration of clipping were analyzed.

Results The incidence of complications was more in Group II (23.5 vs. 8.8\%). There was a reduction in the incidence of complications when the duration of occlusion per episode was reduced; this, however, was not reflected statistically $(p=0.1)$. The total duration of temporary occlusion, when compared, was significantly longer in Group II $(p=0.000)$. The total duration of clipping, however, was not significantly different between those who developed complications and those who did not $(p=0.86)$. The occurrence of complications did not correlate with the vascular territory, number of intermittent clippings, or the total duration of occlusion within their respective

\section{Keywords}

- aneurysm surgery

- temporary occlusion

- elective

- intermittent

- time limit groups.

Conclusion Our study supports the view that the proximal vessel can be safely occluded during aneurysm surgery. The total occlusion period could be safely prolonged when employed in an intermittent fashion with periods of reperfusion to attain the objective of safe dissection. The reduction in duration of each episode of occlusion, though resulted in fewer complications, did not reach statistical significance.
\end{abstract}

\section{Introduction}

Temporary occlusion of the proximal vessel is being increasingly used as an adjunct during aneurysm surgery.
It helps to reduce the pressure within the dome, reduces its size, facilitates safer dissection, and consequently reduces the incidence of intraoperative rupture. Further, in select cases, it aids in evacuating thrombus or atherosclerotic published online March 21, 2015
DOI http://dx.doi.org/ $10.1055 / \mathrm{s}-0035-1549130$ ISSN 2277-954X. (c) 2015 Neurological Surgeons' Society of India
License terms

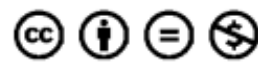


plaque before clipping. Temporary clipping could be either "on demand" as during an intraoperative rupture or used electively. It could be placed continuously or intermittently. Despite its increasing use, there are no clear guidelines regarding its practice. One of the most important parameters is the safe time limit for temporary occlusion. Various studies have attempted to address this concern with no reliable conclusions. Perioperative monitoring techniques, the logical choice to guide the duration of temporary clipping, suffer from a lack of "global monitoring" system. We have been impressed with its usage and practice when used electively in an intermittent fashion. We reviewed our protocol for the last 5 years and analyzed it to provide a practical, safe time period (range) of intermittent occlusion, which is irrespective of the vascular territory in the anterior circulation.

\section{Methods and Patients}

Patients in whom elective, intermittent, temporary clipping protocol was used during 1999 to 2004 were prospectively analyzed. Selection criteria included patients with aneurysms in the anterior circulation. Those patients with multiple aneurysms who underwent temporary occlusion of a single proximal vessel were also included. Exclusion criteria included those with posterior circulation aneurysms and those in whom temporary occlusions of arteries in different vascular territories were done during the course of the same surgery. During the initial years, the proximal vessel occlusion lasted up to an average of 10 minutes per episode, while during the latter period, we reduced the occlusion period to between 5 and 7 minutes. The period of reperfusion was 5 minutes in both protocols. We compared the results of these two groups with respect to the territory involved, number of intermittent clippings, the total duration of occlusion, and the immediate postoperative status of the patient.

\section{Surgical Procedure}

The standard anesthetic protocol was followed as for any aneurysm surgery such as avoiding acute hypertension and achieving adequate brain relaxation. Patients remained normothermic and euvolemic. Prior to temporary occlusion propofol as an infusion and mannitol were started, measures were taken to avoid the anticipated hypotension during propofol infusion. $\mathrm{EtCO}_{2}$ (end tidal carbon dioxide) was kept in the range of 30 to $35 \mathrm{~mm} \mathrm{Hg}$. In general, the temporary clip was applied on the proximal vessel well away from the site of dissection to provide adequate working space, taking care to avoid the perforators. The clip was applied for a fixed period (approximately 5 or 10 minutes) with a 5 -minute reperfusion break between clip applications. Patient's early postoperative clinical status was taken for assessment, as immediate deficit would be reflective of the temporary occlusion. A delayed deficit could be due to other factors such as vasospasm or other postoperative complications. Computed tomography (CT) brain assessment was not a part of the study design. It is included in the results when available and relevant. As a rule, early surgery was preferred; however, some underwent delayed surgery, due to late referral, old age, or a complex aneurysm.

The patients were divided into two groups, Group I, the shorter duration group with a temporary clipping time of less than 7 minutes, and Group II, the longer duration group with a temporary clipping time of about 10 minutes and more. Categorical variables were analyzed using the chisquare test and the continuous variables were analyzed using the Mann-Whitney test and the univariate binary logistic regression. The statistical analysis was performed using SPSS 11.0 (IBM Corporation, Armonk, New York, United States) for windows.

\section{Results}

The total number of patients who satisfied the criteria was 68. There were 34 patients in each of these groups. In Group I among the 34 patients, there were 21 males and 13 females. Age ranged from 28 to 84 years (mean, 57 years). Five patients had multiple aneurysms. There were two intraoperative ruptures, one of them developing a deficit. In Group II, among the 34 patients, there were 16 males and 18 females. Age ranged from 40 to 70 years (mean, 54 years). Here, again five patients had multiple aneurysms. There were eight intraoperative ruptures; one of them developed a deficit.

The details of the artery clipped, number of intermittent clippings, and the total period of clipping with the average are given in -Tables $\mathbf{1}$ and $\mathbf{2}$.

Details of complications in both the groups are given in -Tables 3 and 4. Analysis of complications among the patients in Group I revealed none in the middle cerebral artery (MCA) region. The two patients who developed

Table 1 Details of the artery clipped, number of intermittent clippings, and the total period of clipping in Group I (total no. of patients 34)

\begin{tabular}{|l|l|l|}
\hline $\begin{array}{l}\text { Artery clipped } \\
\text { (no. of patients) }\end{array}$ & No. of intermittent clippings & $\begin{array}{l}\text { Total period of clipping (min) } \\
\text { (average) }\end{array}$ \\
\hline Internal carotid artery (11) & $1-9$ times & $5-44(12.64)$ \\
\hline Anterior cerebral artery (17) & $1-9$ times & $6-43(19.35)$ \\
\hline Middle cerebral artery (6) & $1-7$ times & $5-36(21.83)$ \\
\hline
\end{tabular}


Table 2 Details of the artery clipped, number of intermittent clippings, and the total period of clipping in Group II (total no. of patients 34)

\begin{tabular}{|l|l|l|}
\hline $\begin{array}{l}\text { Artery clipped } \\
\text { (no. of patients) }\end{array}$ & No. of intermittent clippings & $\begin{array}{l}\text { Total period of clipping } \\
\text { (min) (average) }\end{array}$ \\
\hline Internal carotid artery (11) & $10-57(24.18)$ \\
\hline Anterior cerebral artery (16) & $1-6$ times & $18-59(31.44)$ \\
\hline Middle cerebral artery (7) & $2-6$ times & $12-40(27.71)$ \\
\hline
\end{tabular}

deficits among the anterior cerebral artery (ACA) group were elderly and were World Federation of Neurosurgical Societies Grade 3 preoperatively. In one of them the deficit resolved and the $\mathrm{CT}$ did not reveal any infarcts. Other associated factors could have played a major role in the complications. Only two patients, one in the internal cerebral artery (ICA) and one in the ACA group, had deficits and proven infarcts postoperatively.

In Group II, among the MCA group one patient had a low Glasgow Coma Scale score preoperatively and had undergone hematoma evacuation and clipping of the aneurysm emergently in the same sitting with intraoperative rupture. Among the ICA group, one patient had only a lower limb weakness, which resolved by the time of discharge, and the CT did reveal an infarct. The ACA group registered the maximum number of complications. One of the complications was that of a rebleed in the immediate postoperative period and hence it does not reflect the temporary clipping problem. Another patient had an infarct in the posterior temporal and adjacent parietal region suggestive of MCA territory infarct and hence may not reflect the A1-segment clipping.

\section{Statistical Methods}

Statistical analyses were done using SPSS 11.0 for windows and a $p$ value of less than 0.05 was considered significant. Although the incidence of complications was more in Group II (23.5 vs. $8.8 \%$ ), this difference was not statistically significant. It was also found that the numbers of intermittent clippings were not significantly different among patients with complications as compared with those without $(p=0.693)$, which was also supported by univariate binary logistic regression (odds ratio $=0.84 ; 95 \%$ confidence interval 0.56-1.25). Mann-Whitney test was done to assess the total duration of clipping between the two groups. It revealed that the total duration was significantly longer in Group II ( $p$ value $=0.000$ ). The total duration of clipping, however, was not significantly different between those who developed complications and those who did not $(p=0.86)$. The incidence of complications was not associated with the location of the aneurysm $(p=0.9)$.

\section{Discussion}

\section{Temporary Clipping}

The role of temporary clipping in aneurysm microsurgery has been well accepted. ${ }^{1-11}$ Intraoperative rupture is considered the most important and serious complication associated with a grave prognosis with relation to aneurysm surgery. ${ }^{1,3,4,6,8,9,12-17}$ Use of temporary occlusion in reducing its incidence apparently played a major role in improved outcome after aneurysm surgery. ${ }^{1,6,18}$ Studies evaluating the efficacy of temporary clipping have looked into various aspects such as preoperative clinical status, ${ }^{2,10}$ patient profile, ${ }^{2,10}$ relation to timing of surgery, ${ }^{2,7}$ the safe time limit, $4,7,8,10,16,19$ vascular territory, 2,4,7,10,19 cerebral protection measures, 9,10,15,19,20 anesthetic technique, intraoperative monitoring, ${ }^{10,12,14,16,21}$ employing various techniques such as electrophysiological monitoring, $, 8,10,12,16,22$ cortical blood flow, ${ }^{14,21}$ neurochemical monitoring, ${ }^{5,23}$ postoperative clinical assessment, ${ }^{2,4,9,10}$ and postoperative radiological assessment. ${ }^{2,9,10,19}$ Each of these factors has their own limitations.

\section{Postoperative Evaluation}

Two major methods of patient evaluation are clinical and radiological. Clinical evaluation is influenced by various factors such as preoperative clinical status, timing of surgery, retractor injuries, perforator injury, exacerbating vasospasm, venous, or parenchymal injuries, which could cause postoperative deficits. With regard to radiological assessment, all postoperative hypodensities need not be infarcts. They could be postoperative changes or secondary to venous occlusion. ${ }^{10,19}$

\section{Duration of Occlusion}

Studies have reported a wide range of safe occlusion times. $2,4,7-11,14,16,17,21$ Specific arterial territories have been analyzed with regard to the duration. $2,7,10,14,16,19$ In the anterior circulation, MCA territory is thought to be particularly vulnerable to long occlusion periods. It has been shown that the safe period of occlusion could vary even within an individual vascular territory, depending on collateral supply, as within the MCA territory there is large individual variation. ${ }^{7,8,14,19}$ Review of these studies reveals that the safe occlusion period is variable for individuals and vascular territories, as the factors influencing it are manifold. ${ }^{2-5,7,10,17}$ The only consensus is that increasing periods of occlusion lead to increasing chances of infarction. ${ }^{2,10}$

\section{Intermittent versus Continuous}

Temporary clipping could be on demand following intraoperative aneurysm rupture or elective and further it could be continuous or intermittent. There have been 


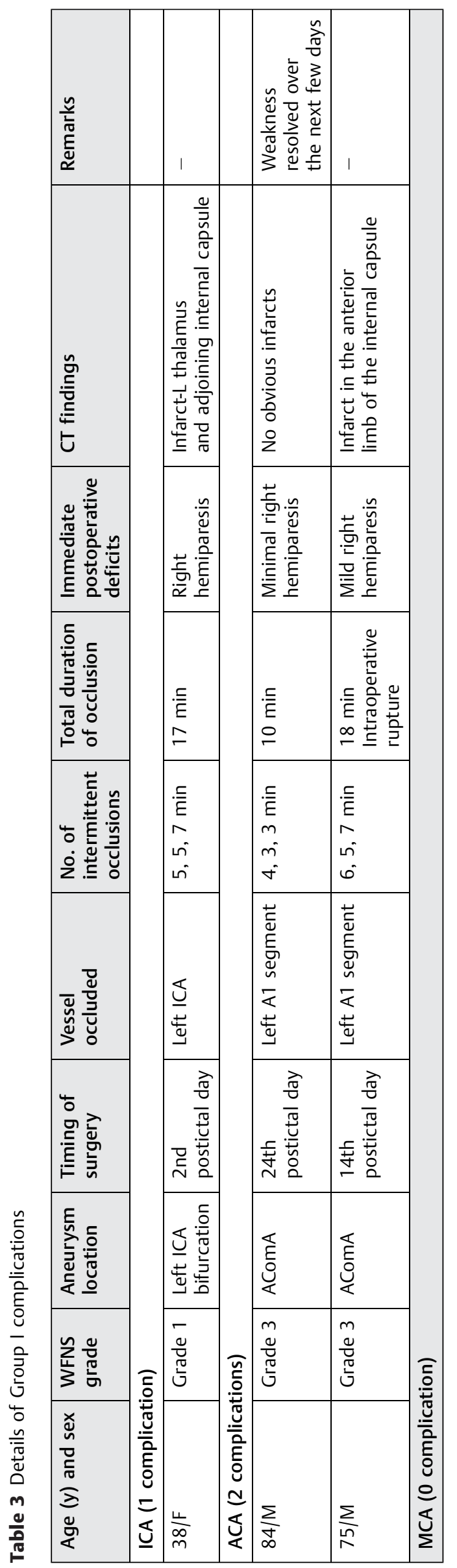

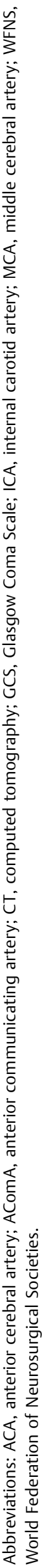

conflicting reports regarding its implications. Some studies have found that continuous occlusion is better than intermittent, and that intermittent occlusion may in fact predispose to ischemia., $90,14,17,24$ On the other hand, studies have also shown that intermittent occlusion with periods of reperfusion appears to be safer than a single episode of continuous occlusion. ${ }^{2,5,17,19}$ Experimental studies also support such a view. ${ }^{17,25-29}$

\section{Our Protocol}

The major complication of temporary clipping is its potential ischemic sequelae. Risk of ischemia during temporary clipping would depend on the following: (1) patient profile, (2) the vessel clipped, (3) characteristics of collateral supply, and (4) duration of clipping. Among these, duration of temporary clipping is the only factor we have control over. Any study trying to focus on one parameter would need to keep all other factors uniform which in the present context would invariably be complicated by a multitude of factors influencing the outcome; hence, we decided to concentrate on evolving a protocol for the safe duration of temporary clipping. ${ }^{10}$

Unlike some studies, ours was a prospective analysis. Based on the available information, one could conclude that shorter the occlusion period, safer would be the procedure. By incorporating intermittent occlusions, one could lengthen the total period of occlusion. Regarding the duration of occlusion, a period of 5 to 7 minutes was considered to be long enough to provide adequate time for dissection and yet short enough to be within the safe range. A range was decided rather than a specific figure, as in practice the actual occlusion period would fall within a range. We planned on a practical protocol in using temporary clipping without considering patient profile, clinical grade, vascular territory, as it would be in a clinical setting, to assess the technique's safety. No monitoring technique was used as it was not available. Anesthetic technique too was standard normotensive, normothermic with administration of propofol and mannitol during temporary occlusion. As mentioned earlier, we relied on the immediate postoperative status of the patient to assess the impact of the temporary clipping, as any delayed neurological deficits could have other causes.

\section{Analysis of Results and Complications}

Between the groups, the total number of patients and their distribution among vascular territories happened to be similar. The overall incidence of complications was less in Group I, validating our hypothesis, though it was not reflected in the statistical analyses, probably due to the small sample size. The total duration of clipping period was longer in Group II and was statistically significant. It must be stated that these patients were also treated during the initial period of the study. Hence, the shorter duration of total period of clipping and increasing experience of the surgeons could have influenced the decreased complications in Group I. Interestingly, the incidences of complications were the 


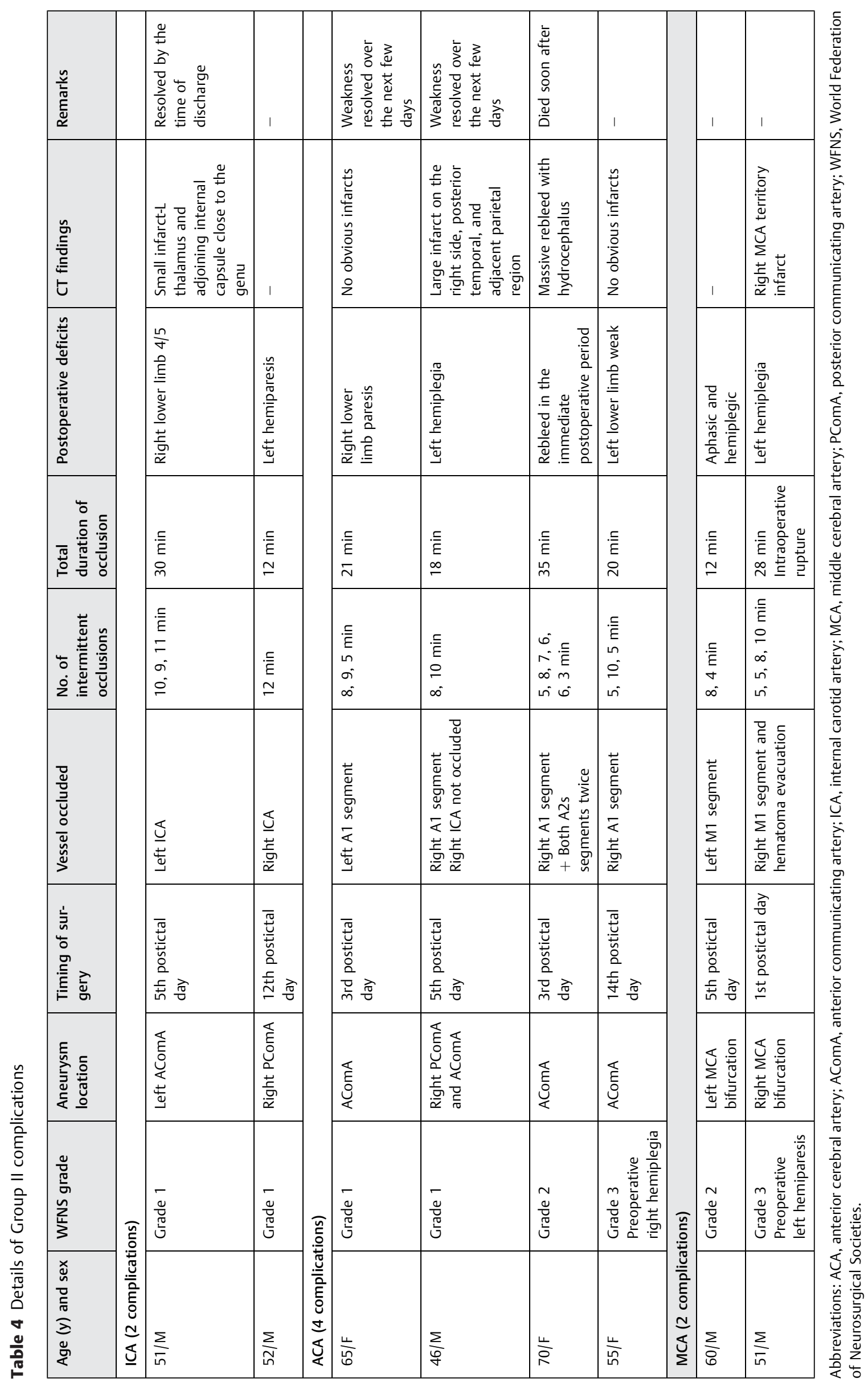


lowest in the MCA territory among both groups, despite the fact that MCA territory is supposedly the most vulnerable to ischemia. The incidence of intraoperative ruptures was more in Group II; however, the complications were not influenced by these events in both the groups. The important observation was that occurrences of complications in both the groups did not correlate with either the total duration of occlusion or the number of intermittent clippings. Other associated factors probably influenced these complications.

In our experience, use of temporary clipping makes the aneurysm dome, soft, compressible, and hence aids in dissection which decreases the chance of a rupture. With a similar protocol, one could go on till the procedure could be completed safely without a finite endpoint. Another interesting observation is that in the immediate postoperative period, there is often a mild paucity of movements on the involved side, which resolves as the patient recovers from anesthesia. We believe from our experience that with short periods of intermittent clipping, one has the dual advantage of more safety and long periods of total occlusion time. 1,2,10 $^{-1}$

In conclusion, elective temporary occlusion of proximal artery appears to be a safe method to employ during aneurysm surgery. When used in an intermittent fashion with periods of reperfusion, the total duration of occlusion can be prolonged to complete the surgical clipping effectively. The reduction in duration of the individual clipping episode resulted in a reduced complications rate, which however did not result in statistical significance. A larger sample size could clarify the issue.

\section{Note}

The work was performed at the Department of Neurosurgery, Indo American Hospital, Brain and Spine Hospital, Chemmanakary, Vaikom, Kerala, India.

\section{Acknowledgments}

The authors thank Prof. V. Rajshekhar for providing editorial assistance and for critically reviewing the manuscript. The authors also thank Mr. Solomon Christopher for helping with the statistical analyses.

\section{References}

1 Batjer H, Samson D. Intraoperative aneurysmal rupture: incidence, outcome, and suggestions for surgical management. Neurosurgery 1986;18(6):701-707

2 Ferch R, Pasqualin A, Pinna G, Chioffi F, Bricolo A. Temporary arterial occlusion in the repair of ruptured intracranial aneurysms: an analysis of risk factors for stroke. J Neurosurg 2002;97(4):836-842

3 Fridriksson S, Säveland H, Jakobsson KE, et al. Intraoperative complications in aneurysm surgery: a prospective national study. J Neurosurg 2002;96(3):515-522

4 Jabre A, Symon L. Temporary vascular occlusion during aneurysm surgery. Surg Neurol 1987;27(1):47-63

5 Kett-White R, Hutchinson PJ, Al-Rawi PG, et al. Cerebral oxygen and microdialysis monitoring during aneurysm surgery: effects of blood pressure, cerebrospinal fluid drainage, and temporary clipping on infarction. J Neurosurg 2002;96(6):1013-1019

6 Leipzig TJ, Morgan J, Horner TG, Payner T, Redelman K, Johnson CS. Analysis of intraoperative rupture in the surgical treatment of 1694 saccular aneurysms. Neurosurgery 2005;56(3):455-468, discussion 455-468

7 Ljunggren B, Säveland H, Brandt L, Kågström E, Rehncrona S, Nilsson PE. Temporary clipping during early operation for ruptured aneurysm: preliminary report. Neurosurgery 1983; 12(5):525-530

8 Mizoi K, Yoshimoto T. Permissible temporary occlusion time in aneurysm surgery as evaluated by evoked potential monitoring. Neurosurgery 1993;33(3):434-440, discussion 440

9 Ogilvy CS, Carter BS, Kaplan S, Rich C, Crowell RM. Temporary vessel occlusion for aneurysm surgery: risk factors for stroke in patients protected by induced hypothermia and hypertension and intravenous mannitol administration. J Neurosurg 1996; 84(5):785-791

10 Samson D, Batjer HH, Bowman G, et al. A clinical study of the parameters and effects of temporary arterial occlusion in the management of intracranial aneurysms. Neurosurgery 1994; 34(1):22-28, discussion 28-29

11 Thomé C, Vajkoczy P, Horn P, Bauhuf C, Hübner U, Schmiedek P. Continuous monitoring of regional cerebral blood flow during temporary arterial occlusion in aneurysm surgery. J Neurosurg 2001;95(3):402-411

12 Buchthal A, Belopavlovic M, Mooij JJA. Evoked potential monitoring and temporary clipping in cerebral aneurysm surgery. Acta Neurochir (Wien) 1988;93(1-2):28-36

13 Giannotta SL, Oppenheimer JH, Levy ML, Zelman V. Management of intraoperative rupture of aneurysm without hypotension. Neurosurgery 1991;28(4):531-535, discussion 535-536

14 Sako K, Nakai H, Kawata Y, Takizawa K, Satho M, Yonemasu Y. Temporary arterial occlusion during anterior communicating or anterior cerebral artery aneurysm operation under tibial nerve somatosensory evoked potential monitoring. Surg Neurol 1998; 49(3):316-322, discussion 322-323

15 Ogilvy CS, Chu D, Kaplan S. Mild hypothermia, hypertension, and mannitol are protective against infarction during experimental intracranial temporary vessel occlusion. Neurosurgery 1996; 38(6):1202-1209, discussion 1209-1210

16 Suzuki J, Yoshimoto T, Kayama T. Surgical treatment of middle cerebral artery aneurysms. J Neurosurg 1984;61(1):17-23

17 Taylor CL, Selman WR, Kiefer SP, Ratcheson RA. Temporary vessel occlusion during intracranial aneurysm repair. Neurosurgery 1996;39(5):893-905, discussion 905-906

18 Cesarini KG, Hårdemark HG, Persson L. Improved survival after aneurysmal subarachnoid hemorrhage: review of case management during a 12-year period. J Neurosurg 1999;90(4): 664-672

19 Lavine SD, Masri LS, Levy ML, Giannotta SL. Temporary occlusion of the middle cerebral artery in intracranial aneurysm surgery: time limitation and advantage of brain protection. J Neurosurg 1997;87(6):817-824

20 Hoffman WE, Charbel FT, Edelman G, Ausman JI. Thiopental and desflurane treatment for brain protection. Neurosurgery 1998; 43(5):1050-1053

21 Ohmoto T, Nagao S, Mino S, et al. Monitoring of cortical blood flow during temporary arterial occlusion in aneurysm surgery by the thermal diffusion method. Neurosurgery 1991;28(1):49-54, discussion 54-55

22 Neuloh G, Schramm J. Monitoring of motor evoked potentials compared with somatosensory evoked potentials and microvascular Doppler ultrasonography in cerebral aneurysm surgery. J Neurosurg 2004;100(3):389-399

23 Jödicke A, Hübner F, Böker DK. Monitoring of brain tissue oxygenation during aneurysm surgery: prediction of procedurerelated ischemic events. J Neurosurg 2003;98(3):515-523 
14 Elective Intermittent Temporary Clipping in Aneurysm Surgery: A Practical Approach Selvapandian et al.

24 Selman WR, Bhatti SU, Rosenstein CC, Lust WD, Ratcheson RA. Temporary vessel occlusion in spontaneously hypertensive and normotensive rats. Effect of single and multiple episodes on tissue metabolism and volume of infarction. J Neurosurg 1994; 80(6):1085-1090

25 David CA, Prado R, Dietrich WD. Cerebral protection by intermittent reperfusion during temporary focal ischemia in the rat. J Neurosurg 1996;85(5):923-928

26 Goldman MS, Anderson RE, Meyer FB. Effects of intermittent reperfusion during temporal focal ischemia. J Neurosurg 1992; 77(6):911-916
27 Iuliano BA, Anderson RE, Meyer FB. Effect of intermittent reperfusion and nitric oxide synthase inhibition on infarct volume during reversible focal cerebral ischemia. J Neurosurg 1995;83(3):491-495

28 Sakaki T, Graf R, Nozaki H, Rosner G, Heiss WD. Possible control of intermittent cerebral ischemia by monitoring of direct-current potentials. J Neurosurg 2001;95(3):495-499

29 Steinberg GK, Panahian N, Sun GH, Maier CM, Kunis D. Cerebral damage caused by interrupted, repeated arterial occlusion versus uninterrupted occlusion in a focal ischemic model. J Neurosurg 1994;81(4):554-559 